Methods Esophageal swallow patterns and refluxes were visually evaluated in preterm and at term bottle fed newborns who underwent Multichannel Intraluminal Impedance (MII) analysis for clinical suspect of GERD.

The swallow frequency, the mean Bolus Head Advance Time (BHAT), the mean Total Bolus Transit Time (TBTT) and reflux characteristics (frequency, duration and impedance bolus exposure index) were assessed for each patient.

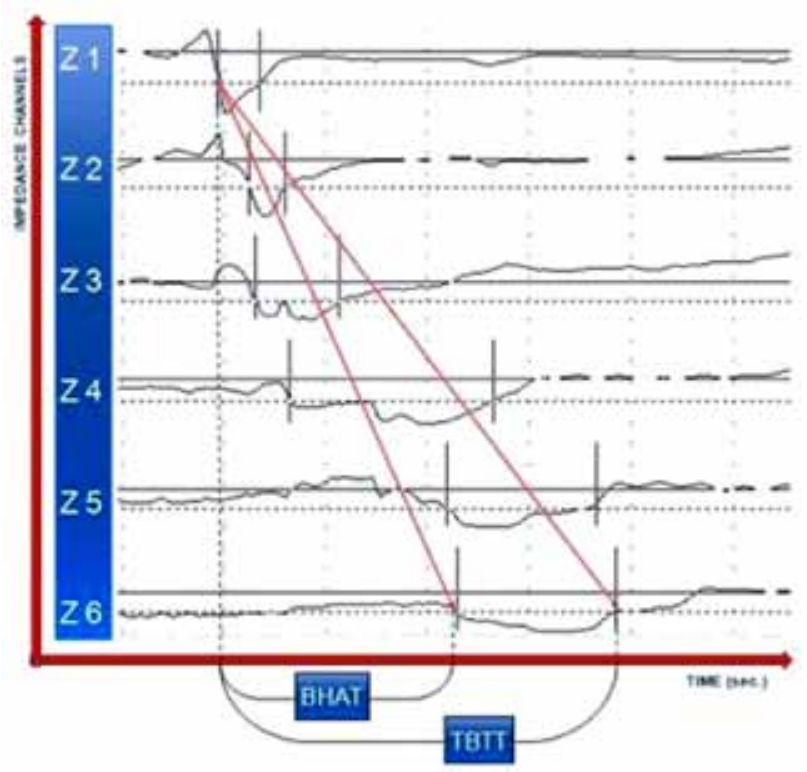

Abstract 714 Figure 1

Pearson correlation was used to evaluate the relationship between swallow and reflux variables; $p$ was set at 0.05 .

Results Fifty-four newborns (10 preterm) were enrolled in the study. They had a median(IOR) age of 22(22.5) days, a Gestational age of 37(6) weeks and a postconceptional age of 41.8(4.9) weeks. A significant positive correlation was found between TBTT and IBEI $\%(r=0.494 ; p<0.001)$ and between TBTT and reflux duration $(\mathrm{r}=0.321 ; \mathrm{p}=0.018)$.

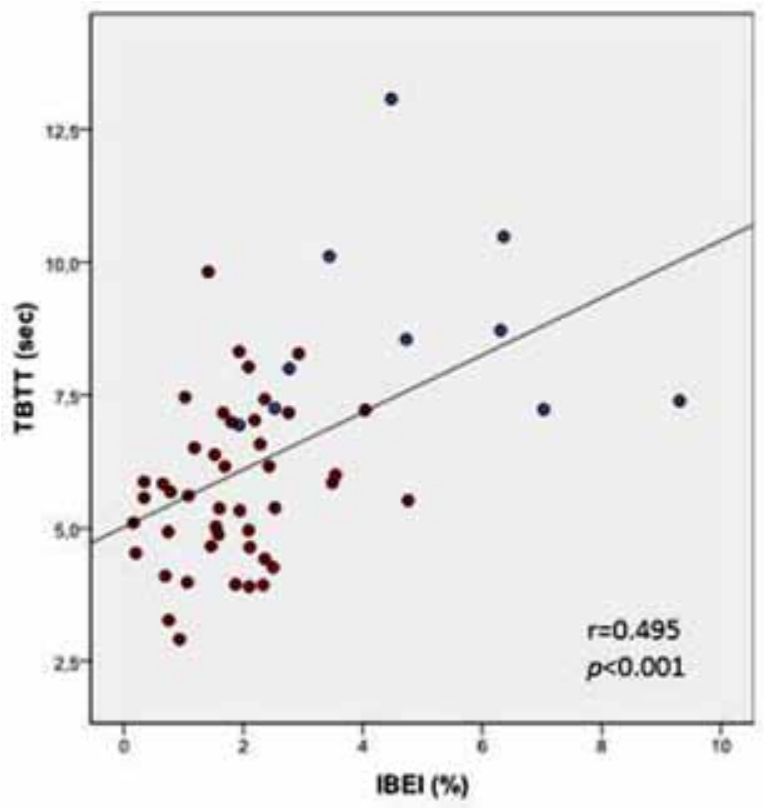

Abstract 714 Figure 2
Conclusions MII is a valid technique to evaluate esophageal bolus transit and refluxes. Our data support that esophageal motor dysfunction due to immaturity affect esophageal swallowing patterns and esophageal clearance time in GERD newborns.

\section{DENTAL EROSION AND GASTROESOPHAGEAL REFLUX DISEASE (GERD) IN CHILDREN}

doi:10.1136/archdischild-2012-302724.0715

${ }^{1} \mathrm{~F}$ Javadzadeh, ${ }^{2} \mathrm{M}$ Rafeey. 'Dentistry, Tabriz University of Medical Sciences, Aras

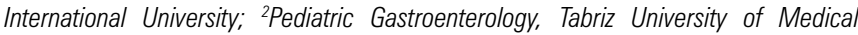
Sciences, Liver \& Gastrointestinal Research Center, Tabriz, Iran

Background Dental erosion is a complication of gastroesophageal reflux Disease (GERD) in adults; in children, it is not clear if GER has a role in dental pathologic conditions. Dietary intake, oral hygiene, high bacterial capacity, and decreased salivary flow might contribute individually to GERD development or dental erosion, but their potential involvement in dental erosion from GER is not agreed. We investigated the prevalence of dental erosion among children with and without GER symptoms, and whether salivary flow rate to location-specific dental erosion

Methods We performed a cross-sectional study of 40 children (ages,3-6 y) with symptoms of GERD and 30 asymptomatic children (controls); all completed a questionnaire on dietary exposure. Teeth were examined for erosion into dentin, erosion locations, and affected surfaces. All subjects responded a detailed frequency questionnaire related to acidic drinks, foods, and sugar consumption and participated in a clinical dental examination. The caries experience of the children was recorded according to World Health Organization criteria, and erosion was scored according to the Eccles and Jenkins grading scale.

Results This Survey showed that the prevalence of erosion on palatal surfaces of the primary teeth was $42 \%$ in 3-6-year-olds with GERD. This finding to be significantly higher than for healthy subjects $(\mathrm{P}<0.05)$. The salivary flow rate, and frequency of acidic drinks, foods, and sugar consumption were found to be similar in both groups.

Conclusion This current investigation has showed that GERD children were at an increased threat of developing erosion and caries compared with healthy subjects.

\section{DOES BLOODY ASPIRATE REFLECT THE STATE OF UPPER GASTROINTESTINAL MUCOSA IN A CRITICALLY ILL NEWBORN?}

doi:10.1136/archdischild-2012-302724.0716

R Tomerak, AA El Badawy, AE Eskander, AH Mahmoud. Cairo University, Cairo, Egypt

Background and study aims Critically ill newborns have many risk factors to develop stress related mucosal lesions (SRML). We used upper endoscopy to evaluate the presence of SRML in these neonates, to know the specificity and sensitivity of the bloody gastric aspirate to detect SRML and to identify the risk factors associated with the presence of SRML and bloody gastric aspirate.

Patients and methods This is a cross-sectional study done on 100 critically ill newborn after becoming clinically stable. SRML were diagnosed if there is hyperemia, erosions or ulcers in the esophagus, stomach, and/or the duodenum. The association between the various clinico-laboratory findings and the presence of SRML and bloody gastric aspirate were studied.

Results SRML were found in $77 \%$ of neonates in the NICU though frank bloody aspirate was detected in only $22 \%$ of neonates. The presence of bloody aspirate showed low sensitivity (24.68\%) for the presence of SRML and high specificity (86.96\%). The presence of bloody gastric aspirate showed a double fold risk for the presence SRML $(\mathrm{OR}=2.184, \mathrm{CI}=0.584-8.171)$. Factors associated with SRML 